\title{
A rapid, simultaneous and quantitative analysis of 26 ginsenosides in white and red Panax ginseng using LC-MS/MS
}

\author{
Junghak Lee ${ }^{1}$, Heeju Han' ${ }^{1}$, Xiu Yuan ${ }^{1}$, Eunyoung Park' ${ }^{1}$ Jonghwa Lee ${ }^{2^{*}}$ and Jeong-Han Kim ${ }^{1 *}$ (i)
}

\begin{abstract}
A quantitative analysis of ginsenoside is very important for ginseng studies because each ginsenoside shows different medical activity and metabolic pathway. In this study, a rapid, simultaneous, and quantitative analysis of 26 ginsenosides (Rb1, Rb2, Rc, Rd, Re, Rf, Rg1, Rg2(R), Rg2(S), Rg3(S), Rg3(R), Rg5, Rg6, Rh1(R), Rh1(S), Rh2(R), Rh2(S), F1, F2, F3, F4, $\mathrm{K}, \mathrm{Mc}, \mathrm{PPT}(\mathrm{S}), \mathrm{XVII}$, and $\mathrm{Y}$ ) in white, and red Panax ginseng was established using multiple reaction monitoring (MRM) mode on ultra-high-performance liquid chromatography-tandem mass spectrometry (UHPLC-MS/MS). The mobile phase of water and methanol containing $0.1 \%$ formic acid and HSS T3 C18 analytical column was used for the chromatographic separation. The four sets of stereoisomers were successfully separated within a 26-min run time, eluting the S-isomer faster than the R-isomer with higher concentration. The ginseng extract was diluted by 100, 400 and 8000 times to fit in the calibration range and quantitated by the standard addition method. Matrix matched calibration by mixing $64 \mu \mathrm{L}$ of the ginseng extract with $16 \mu \mathrm{L}$ of the standard solution was used for compensating the matrix effect. Such quantitation methodology using dilution, standard addition and matrix matching resulted in precise and unambiguous quantitation of 26 ginsenosides in ginseng products. Major ginsenosides were observed at relatively higher concentrations in red Panax ginseng and the Mc was detected and quantitated for the first time in this study. The comprehensive quantitation system established in this study will contribute to quality evaluation, breeding and culturing, and quantitative metabolomics study of ginseng.
\end{abstract}

Keywords: Ginsenoside, Red Panax ginseng, Simultaneous analysis, UHPLC-MS/MS, White Panax ginseng

\section{Introduction}

The Korean ginseng belonging to Panax, family Araliaceae has been known as medicinal herbs and used for functional foods and dietary supplements which can provide various benefits such as immunity-boosting, antiinflammatory, antioxidative activity, fatigue recovery, blood flow improvement, and memory improvement [1, 2]. For these reason, many researches identified the active ingredient known as ginsenoside and isolated by various

\footnotetext{
*Correspondence: jhlee006@gmail.com; kjh2404@snu.ac.kr

1 Department of Agricultural Biotechnology and Research Institute

of Agriculture and Life Sciences, Seoul National University, Seoul 08826, Republic of Korea

${ }^{2}$ Center for Food and Drug Analysis, Ministry of Food and Drug SafetyGyeongin Regional Office, Incheon 22133, Republic of Korea
}

analytical techniques such as TLC, GC, HPLC, capillary electrophoresis, and near infra-red spectroscopy [3]. Although simultaneous quantitation of ginsenoside was attempted by HPLC-UVD [4] and UHPLC-MS/MS [5], the HPLC method not only had low sensitivity but also had limited number of target compounds due to the overlapping of compounds. Since multiple reaction monitoring (MRM) mode allows the overlapping of retention time for each compound, the MS/MS with triple quadrupole instrument has widely been used for rapid and simultaneous analysis of many types of compounds. For profiling purposes, UHPLC-MS/MS has been generally used to compare the relative content of ginsenoside [6, 7]. Because the medical activity of ginsenosides depends on its quantities in ginseng or its formulation, a precise 
quantitation is very critical for evaluating of the medical activity. In this study, a rapid and simultaneous analysis of 26 ginsenosides (Rb1, Rb2, Rc, Rd, Re, Rf, Rg1, Rg2(R), Rg2(S), Rg3(S), Rg3(R), Rg5, Rg6, Rh1(R), Rh1(S), Rh2(R), Rh2(S), F1, F2, F3, F4, K, Mc, PPT(S), XVII, and Y) was achieved quantitatively with 26 min of chromatographic running time using MRM mode on UHPLC-MS/MS, including chromatographic separation of isomeric ginsenosides. To compensate the matrix effect in the UHPLCMS/MS detection, matrix matched calibration was used and standard addition method was applied for precise quantitation. Various types of columns and elution solvents were compared for efficient, rapid and simultaneous separation of 26 ginsenosides. The UHPLC-MS/MS method developed was successfully applied to quantitation of those in commercial white Panax ginseng (WG) and red Panax ginseng (RG).

\section{Materials and methods \\ Chemicals and reagents}

Twenty-six reference standards (purity $\geq 98.0 \%$ ) of ginsenosides (Rb1, Rb2, Rc, Rd, Re, Rf, Rg1, Rg2(R), Rg2(S), Rg3(R), Rg3(S), Rg5, Rg6, Rh1(R), Rh1(S), Rh2(R), Rh2(S), F1, F2, F3, F4, K, Mc, XVII, Y, and PPT(S)) were purchased from AMBO (Ambo institute, Seoul, Korea). HPLC grade methanol was purchased from Merck (Darmstadt, Germany) while LC-MS grade of methanol and formic acid were obtained from Merck (Darmstadt, Germany) and Sigma-Aldrich (St. Louis, Mo, USA), respectively.

\section{White and red Panax ginseng}

Six-year old white and red Panax ginseng root, cultivated in Geumsan-gun, Chungcheongnam-do, Rerpublic of Korea, were purchased from the commercial market.

\section{Analytical standard solution}

Individual stock solutions of 26 reference standards were prepared in methanol at the concentration of $1,000 \mu \mathrm{g} /$ $\mathrm{mL}$ and working standard solutions with the concentrations of $0.01,0.02,0.05,0.1,0.2,0.5,1,2,5,10,20$, and $50 \mu \mathrm{g} / \mathrm{mL}$ were prepared by dilution with methanol.

\section{Selection of LC mobile phase and MRM optimization in UHPLC-MS/MS}

UHPLC-MS/MS analysis was carried out on a Shimadzu LC-MS 8040 triple quadrupole mass spectrometer (Kyoto, Japan). Electrospray ionization (ESI) mode was used with de-solvation temperature of $250{ }^{\circ} \mathrm{C}$ and heat block temperature of $400{ }^{\circ} \mathrm{C}$. The flow rate of nitrogen for nebulizing and drying gas was $3 \mathrm{~L} / \mathrm{min}$ and $15 \mathrm{~L} / \mathrm{min}$, respectively.
The intensity of precursor ion was obtained in positive and negative full scan mode $(50 \sim 1,200 \mathrm{~m} / \mathrm{z})$ by injecting of $5 \mu \mathrm{L}$ standard working solution $(10 \mu \mathrm{g} / \mathrm{mL})$. Two different mobile phase modifiers of $0.1 \%$ formic acid in water and $5 \mathrm{mM}$ ammonium formate in water were compared as an UHPLC-MS/MS eluent under the ratio of 50:50 with methanol. The optimal MRM transitions were obtained, using $0.1 \%$ formic acid in water/methanol as UHPLC-MS/MS eluent, by dissociation of selected the precursor ions to produce quantifier and qualifier ions (Table 1).

\section{Separation of 26 ginsenosides}

To separate 26 ginsenosides, four kinds of C18 UHPLC columns such as kinetex $(2.1 \times 100 \mathrm{~mm}, 2.6 \mu \mathrm{m}$, Phenomenex, Torrance, CA, USA), Luna $(2.0 \times 100 \mathrm{~mm}, 3 \mu \mathrm{m}$, Phenomenex, Torrance, CA, USA), BEH (2.1 x100 mm, $1.7 \mu \mathrm{m}$, Waters, Milford, MA, USA), and HSS T3 C18 ( $2.1 \times 150 \mathrm{~mm}, 1.8 \mu \mathrm{m}$, Waters, Milford, MA, USA) were compared with $0.1 \%$ formic acid in water (mobile phase A) and methanol (mobile phase B) as mobile phase. The gradient program used for the comparing was as follows: $0-1 \min (50 \% \mathrm{~B}) ; 1-45 \mathrm{~min}(50-95 \% \mathrm{~B}) ; 45-50 \mathrm{~min}$ (95\% B); 50-50.1 min (95-50\% B); $50.1-60 \min (50 \% \mathrm{~B})$. In addition, shorter HSS T3 C18 column $(2.1$ x $50 \mathrm{~mm}$, $1.8 \mu \mathrm{m}$, Waters, Milford, MA, USA) was further tested to minimize analytical time, using the following gradient program: $0-0.1 \mathrm{~min}(30 \% \mathrm{~B})$; $0.1-1 \mathrm{~min}(30-50 \% \mathrm{~B})$; $1-22 \mathrm{~min}(50-88 \% \mathrm{~B}) ; 22-23 \mathrm{~min}(88 \% \mathrm{~B}) ; 23-23.1 \mathrm{~min}$ $(30 \% \mathrm{~B}) ; 23.1-26 \mathrm{~min}(30 \% \mathrm{~B})$ with the flow rate of $0.3 \mathrm{~mL} / \mathrm{min}$.

\section{Sample preparation for ginsenosides analysis}

Six year-old white and red Panax ginseng roots were freeze-dried for 3 days in a Freeze-Dryer (Il-Shin Lab Tech. Co., Korea), and pulverized by a ball-mill instrument (MM 400, Retsch, GmbH, Haan, Germany). Fifty milligrams of each ginseng powder were extracted with $1 \mathrm{~mL}$ of $70 \%$ methanol in an ultra-sonic bath for $30 \mathrm{~min}$ at $30^{\circ} \mathrm{C}$ followed by centrifugation at $15,000 \mathrm{rpm}$ at $4{ }^{\circ} \mathrm{C}$. The supernatant was used for quantitation.

\section{Quantitation by standard addition method}

An aliquot $(64 \mu \mathrm{L})$ of ginseng extract without dilution was used as quantitation group I (QG-I), and another aliquot was diluted by 100 (QG-II), 400(QG-III), and 8000 times (QG-IV) with 70\% methanol, respectively. Each of QG-I, -II, -III, and -IV were spiked with working standard solution $(16 \mu \mathrm{L})$ for standard addition quantitation. Five microliter of QG-I, -II, -III, and -IV was injected and quantitation was performed by calculating $\mathrm{x}$-intercept value when $\mathrm{y}=0$ of calibration curve equation $\mathrm{y}=\mathrm{ax}+\mathrm{b}$. In white Panax ginseng, QG-I was applied for ginsenoside 
Table 1 Optimized MRM transitions of ginsenosides in UHPLC-MS/MS analysis

\begin{tabular}{|c|c|c|c|c|c|c|}
\hline \multirow[t]{2}{*}{ No. } & \multirow[t]{2}{*}{ Ginsenoside } & \multirow[t]{2}{*}{ Monoisotopic mass } & \multirow[t]{2}{*}{ Adduct formula } & \multicolumn{3}{|c|}{ MRM transitions } \\
\hline & & & & Precursor & Quantifier & Qualifier \\
\hline 1 & $\operatorname{Re}$ & 946.55 & {$[\mathrm{M}+\mathrm{Na}]^{+}$} & 969.40 & 789.55 & 203.05 \\
\hline 2 & $\mathrm{Rg} 1$ & 800.49 & {$[\mathrm{M}+\mathrm{Na}]^{+}$} & 823.50 & 643.30 & 203.05 \\
\hline 3 & $\operatorname{Rh} 1(S)$ & 638.44 & {$[\mathrm{M}+\mathrm{Na}]^{+}$} & 661.30 & 203.10 & 22.95 \\
\hline 4 & F3 & 770.48 & {$[\mathrm{M}+\mathrm{Na}]^{+}$} & 793.30 & 335.10 & - \\
\hline 5 & $\mathrm{Rh} 1(\mathrm{R})$ & 638.44 & {$[\mathrm{M}+\mathrm{Na}]^{+}$} & 661.30 & 22.90 & 203.05 \\
\hline 6 & $\mathrm{~F} 1$ & 638.44 & {$[\mathrm{M}+\mathrm{Na}]^{+}$} & 661.20 & 203.10 & 481.30 \\
\hline 7 & $\mathrm{Rb} 1$ & 1108.60 & {$[\mathrm{M}+\mathrm{Na}]^{+}$} & 1131.50 & 365.00 & 789.35 \\
\hline 8 & $\mathrm{Rc}$ & 1078.59 & {$[\mathrm{M}+\mathrm{Na}]^{+}$} & 1101.50 & 335.00 & 789.35 \\
\hline 9 & $\mathrm{Rb} 2$ & 1078.59 & {$[\mathrm{M}+\mathrm{Na}]^{+}$} & 1101.50 & 789.35 & 335.05 \\
\hline 10 & PPT(S) & 476.39 & {$\left[\mathrm{M}-2 \mathrm{H}_{2} \mathrm{O}+\mathrm{H}\right]^{+}$} & 441.30 & 43.35 & 123.05 \\
\hline 11 & $\mathrm{Rd}$ & 946.55 & {$[\mathrm{M}+\mathrm{Na}]^{+}$} & 969.40 & 789.35 & 203.05 \\
\hline 12 & $X V I I$ & 946.55 & {$[\mathrm{M}+\mathrm{Na}]^{+}$} & 969.40 & 365.10 & - \\
\hline 13 & $\operatorname{Rg} 3(S)$ & 784.50 & {$\left[\mathrm{M}-2 \mathrm{H}_{2} \mathrm{O}+\mathrm{H}\right]^{+}$} & 749.50 & 407.35 & 163.10 \\
\hline 14 & $\operatorname{Rg} 3(\mathrm{R})$ & 784.50 & {$\left[\mathrm{M}-2 \mathrm{H}_{2} \mathrm{O}+\mathrm{H}\right]^{+}$} & 749.50 & 163.10 & 407.35 \\
\hline 15 & $\mathrm{~F} 2$ & 784.50 & {$[\mathrm{M}+\mathrm{Na}]^{+}$} & 807.50 & 627.30 & 203.10 \\
\hline 16 & Mc & 754.49 & {$[\mathrm{M}+\mathrm{Na}]^{+}$} & 777.60 & 335.10 & 336.10 \\
\hline 17 & K & 622.44 & {$[\mathrm{M}+\mathrm{Na}]^{+}$} & 645.20 & 203.00 & 465.30 \\
\hline 18 & $\operatorname{Rh} 2(S)$ & 622.44 & {$\left[\mathrm{M}-2 \mathrm{H}_{2} \mathrm{O}+\mathrm{H}\right]^{+}$} & 587.40 & 407.30 & 123.10 \\
\hline 19 & $\operatorname{Rh} 2(\mathrm{R})$ & 622.44 & {$\left[\mathrm{M}-2 \mathrm{H}_{2} \mathrm{O}+\mathrm{H}\right]^{+}$} & 587.40 & 407.35 & 109.10 \\
\hline 20 & $\mathrm{Rf}$ & 800.49 & {$[\mathrm{M}-\mathrm{H}]^{-}$} & 799.50 & 475.45 & 100.90 \\
\hline 21 & $\operatorname{Rg} 2(S)$ & 784.50 & {$[\mathrm{M}-\mathrm{H}]^{-}$} & 783.50 & 475.35 & 161.15 \\
\hline 22 & $\operatorname{Rg} 2(R)$ & 784.50 & {$[\mathrm{M}-\mathrm{H}]^{-}$} & 783.50 & 475.45 & 119.10 \\
\hline 23 & Rg6 & 766.49 & {$[\mathrm{M}-\mathrm{H}]^{-}$} & 765.50 & 101.10 & 161.10 \\
\hline 24 & $\mathrm{~F} 4$ & 766.49 & {$[\mathrm{M}-\mathrm{H}]^{-}$} & 765.50 & 101.10 & 161.10 \\
\hline 25 & Y & 754.49 & {$[\mathrm{M}-\mathrm{H}]^{-}$} & 753.50 & 149.15 & 89.05 \\
\hline 26 & $\operatorname{Rg} 5$ & 766.49 & {$[\mathrm{M}-\mathrm{H}]^{-}$} & 765.50 & 101.05 & 161.20 \\
\hline
\end{tabular}

F1, F2, F3, K, Mc, PPT(S), Rg1, Rh2(R, S), XVII, and Y, while QG-II for F4, Rg2(R, S) and Rg6. QG-III was for Rd, Rf, Rg3(R, S), Rg5 and Rh1(R, S) and QG-IV was for $\mathrm{Rb} 1, \mathrm{Rb} 2, \mathrm{Rc}$ and Re. In red Panax ginseng, QG-I was applied for ginsenoside F1, F2, F3, K, Mc, PPT(S), Rh2(R, S), XVII, and Y, while QG-II for F4, Rg2(R, S), and Rg6. QG-III was for Rd, Rf, Rg3(R, S), Rg5, and Rh1(R, S), and QG-IV was for Rb1, Rb2, Rc, Re, and Rg1.

\section{Results and discussion}

Selection of LC mobile phase and optimization of the MRM transitions

Since ion adduct types and intensity tend to rely on the mobile phase modifier such as formic acid or ammonium formate for ESI of UHPLC-MS/MS [8], 0.1\% formic acid in water and $5 \mathrm{mM}$ ammonium formate in water, were tested to check for the adduct types and intensity of the precursor ion on MS scan mode. In positive scan mode, most precursor ions of ginsenosides showed better intensity in the water with $0.1 \%$ formic acid than in the water with $5 \mathrm{mM}$ ammonium formate. In addition to the low intensity in $5 \mathrm{mM}$ ammonium formate, the precursor ions were not even generated for F4, Rb1, Rb2, Rc, Re, Rg5, and XVII. The most of ginsenosides produced sodium adduct precursor ion $[\mathrm{M}+\mathrm{Na}]^{+}$as a precursor ion while $\mathrm{PPT}(\mathrm{S}), \operatorname{Rg} 3(\mathrm{R})$, $\mathrm{Rg} 3(\mathrm{~S}), \mathrm{Rh} 2(\mathrm{R})$, and $\mathrm{Rh} 2(\mathrm{~S})$ showed $\left[\mathrm{M}+\mathrm{H}-2 \mathrm{H}_{2} \mathrm{O}\right]^{+}$ precursor ion as also reported in literature [9]. In negative mode, both formic acid and ammonium formate resulted in similar intensity, for $\mathrm{Rf}, \operatorname{Rg} 2(\mathrm{R}, \mathrm{S}), \operatorname{Rg} 6, \mathrm{~F} 4$, $\mathrm{Y}$, and $\mathrm{Rg} 5$ producing $[\mathrm{M}-\mathrm{H}]^{-}$precursor ion. Their intensities were higher than sodium adducted precursors $[\mathrm{M}+\mathrm{Na}]^{+}$from the positive mode. Therefore, the mobile phase for the MRM optimization was chosen to water containing $0.1 \%$ formic acid (A) and methanol (B) as UHPLC-MS/MS eluent. After collision induced dissociation of precursor ions, the product ion providing the highest intensity was selected as the quantifier ion while the second highest product ion was selected as the qualifier ion (Table 1, Additional file 1: Fig. S1). 


\section{Separation of 26 ginsenosides including isomers} on various analytical columns

It is very important to quantitate the constitutional and stereisomeric isomer of ginsenosides independently because each isomer was known to have different activity [10-12]. Analysis of ginsenoside isomers required the derivatization for GC analysis [13] or 2-dimensional UHPLC-MS/MS for the separation [14]. Separating isomers using MS detection is challenging due to the similarity of the MS spectra and MRM transition between isomers. Therefore, chromatographic separation is required to distinguish the isomers. In this study, we tried to resolve the isomers chromatographically using the C18 column which is commonly used in UHPLCMS/MS analysis.

Three common C18 columns were tested first because they have widely been used for the analysis of ginsenosides [3, 15-18]. The stereoisomer of ginsenosides, $\mathrm{Rh} 2(\mathrm{R}, \mathrm{S})$ and $\operatorname{Rg} 3(\mathrm{R}, \mathrm{S})$ were not separated on those three columns, instead, the HSS T3 C18 column (2.1 x
$150 \mathrm{~mm}, 1.8 \mu \mathrm{m}$, Waters, Milford, MA, USA) gave a very good resolution for $\operatorname{Rg} 3(\mathrm{~S}) / \operatorname{Rg} 3(\mathrm{R})$, and $\mathrm{Rh} 2(\mathrm{~S}) / \mathrm{Rh} 2(\mathrm{R})$ (Fig. 1), resulting in the successful separation of 26 ginsenosides within $60 \mathrm{~min}$. However, the analytical running time of $60 \mathrm{~min}$ with the column of $150 \mathrm{~mm}$ length was considered to be quite long, so shorter HSS T3 C18 column of $50 \mathrm{~mm}$ length was tried again to analyze in 26 min, saving 34 min of analytical time (Fig. 2b).

\section{Quantitation of $\mathbf{2 6}$ ginsenosides in red and white Panax ginseng using matrix matching and standard addition method}

In HPLC-UVD analysis of ginsenosides, solvent standard solutions have been used for quantitation as the external calibration method thanks to the lack of matrix effect [19]. However, in the UHPLC-MS/MS analysis, the matrix-matched calibration to compensate the matrix effects by a co-extracted matrix, which is a general and inevitable phenomenon, is an essential procedure for precise calibration and quantitation [8]. Although several

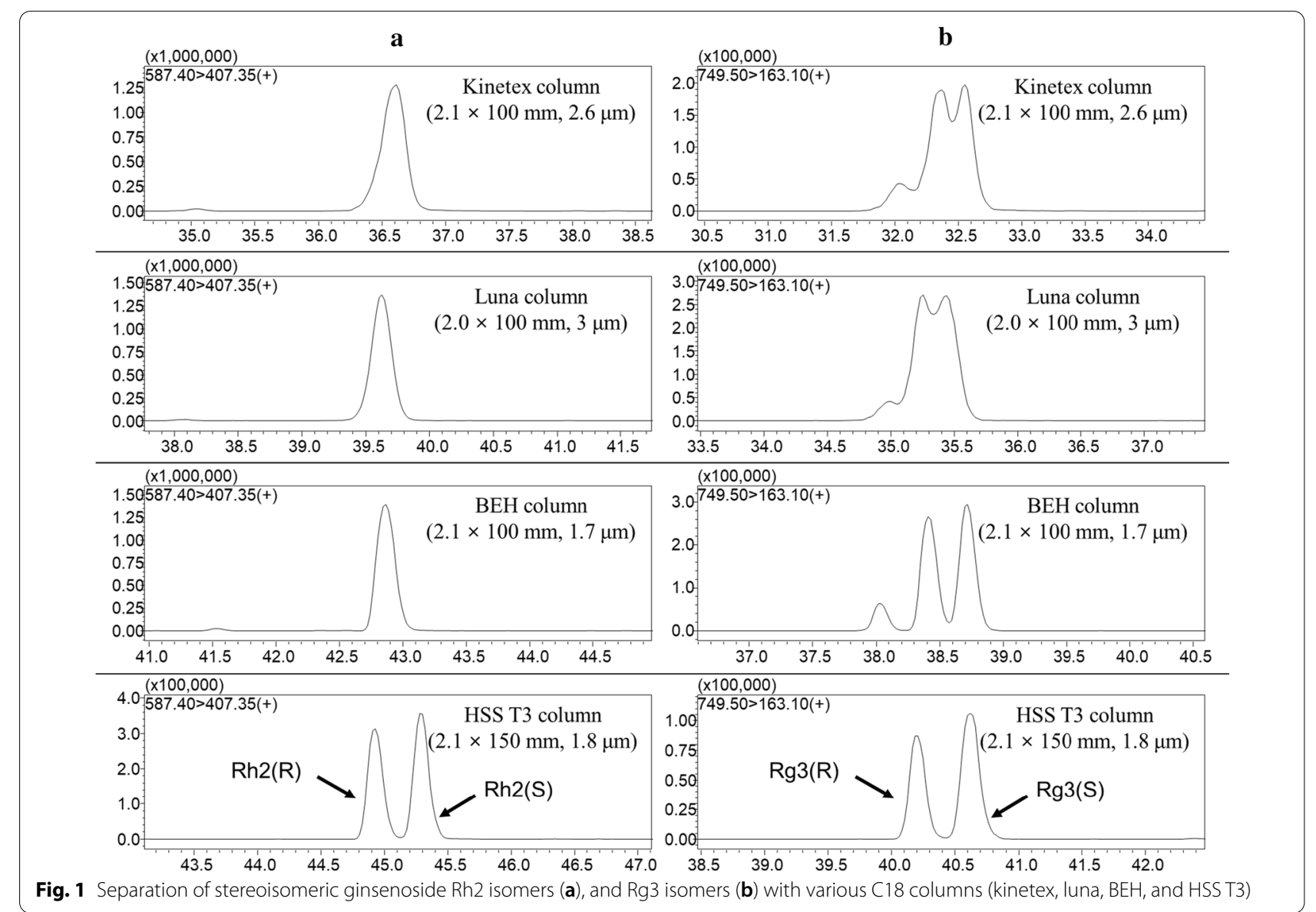



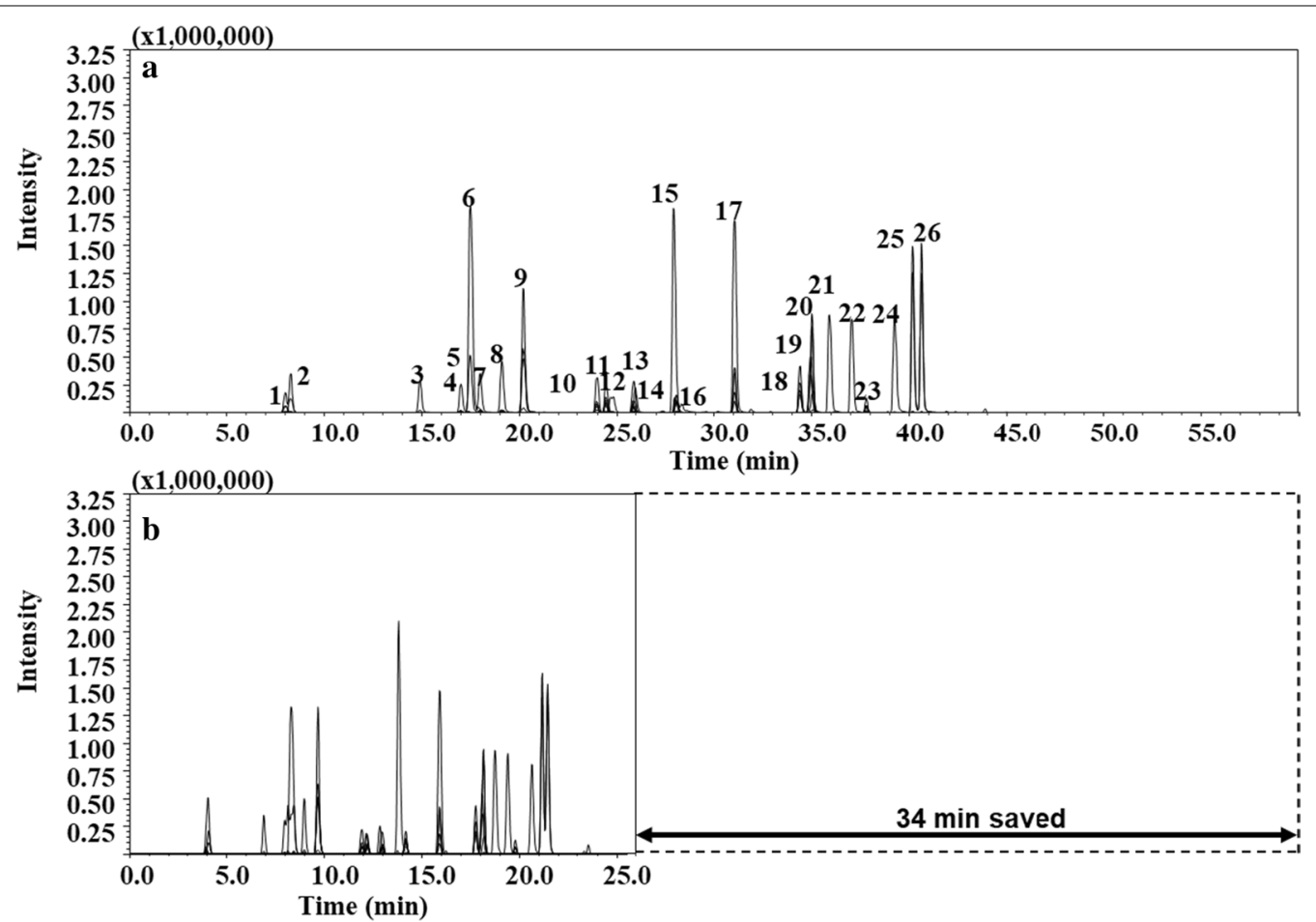

Fig. 2 UHPLC-MS/MS TIC chromatogram of 26 ginsenosides on HSS T3 C18 column $(2.1 \times 150$ mm, $1.8 \mu \mathrm{m})(\mathbf{a})(1 ; \mathrm{Re}, 2 ; \mathrm{Rg} 1,3 ; \mathrm{Rf}, 4 ; \mathrm{Rg} 2(\mathrm{~S}), 5 ; \mathrm{F} 3,6$; $\operatorname{Rh} 1(\mathrm{~S}), 7 ; \operatorname{Rg} 2(\mathrm{R}), 8 ; \operatorname{Rh} 1(\mathrm{R}), 9 ; \mathrm{F1}, 10 ; \mathrm{Rb} 1,11 ; \mathrm{Rc}, 12 ; \mathrm{Rb} 2,13 ; \mathrm{F} 4,14 ; \mathrm{Rg} 6,15 ; \mathrm{Rd}, 16 ; \mathrm{PPT}(\mathrm{S}), 17 ; \mathrm{XVII}, 18 ; \mathrm{Rg} 3(\mathrm{~S}), 19 ; \mathrm{Rg} 3(\mathrm{R}), 20 ; \mathrm{F2}, 21 ; \mathrm{Mc}, 22 ; \mathrm{Y}, 23 ; \mathrm{Rg} 5$, 24; K, 25; Rh2(S), 26; Rh2(R)), HSS T3 C18 column (2.1 x $50 \mathrm{~mm}, 1.8 \mu \mathrm{m})(\mathbf{b})$

studies used UHPLC-MS/MS quantitation, their methodology for the matrix matching or the quantitation was not described in detail $[5,18,20]$.

The endogenous concentrations of ginsenosides in sample extracts that are already present in the extract makes the quantitation difficult since the external standard calibration using the solvent standard cannot be applicable for the quantitation. Therefore, we decided to use the standard addition method for precise calibration and quantitation. SANTE/11813/2017 guideline [21] suggested a standard addition method in which calibration curve range is in less than 5 times of the area of original level to prevent distortion of the calibration curve. Another problem is the large difference of each ginsenoside concentration in ginseng, ranging from a few $\mathrm{mg} /$ $\mathrm{kg}$ to several thousands of $\mathrm{mg} / \mathrm{kg}$, which means high fold dilution is needed to fit in the calibration range. Therefore, to fit the ginsenoside of higher concentration into the calibration range of MS/MS, original extract (QGI) was diluted to make QG-II (100 times dilution), -III (400 times dilution) and -IV (8000 times dilution) and standard solution of various concentration was added for quantitation with standard addition method.
The matrix matched calibration was performed by mixing $64 \mu \mathrm{L}$ of the ginseng extract and $16 \mu \mathrm{L}$ of the working standard solution. Three or four point of calibration was applied as recommended by SANTE/11813/2017 [21]. Highly correlated equations were obtained for each ginsenoside (Table 2; Fig. 3) from white and red Panax ginseng samples to calculate ginsenoside contents in precise way.

In white Panax ginseng, major ginsenoside of Rb1, Rb2, Rc, Rd, Re, Rf, and Rg1 [22] were observed at relatively higher concentration as expected [5, 23, 24]. The S-isomer was eluted faster than the R-isomer with the higher concentration $[6,20]$. Among other minor ginsenoside, Mc and $Y$ were analyzed for the first time in this study, and Mc was quantitated. Rg2(S) was observed at higher concentration compared to other reports. Most of the ginsenoside in white Panax ginseng were observed in red Panax ginseng with higher concentration than white Panax ginseng $[5,23,25]$. Major ginsenoside in red Panax ginseng in this study contained higher amount than that of reported literature [5, 24, 25]. Compound PPT(S) and Y were not detected in both of white and red Panax ginseng. 
Table 2 Concentration of ginsenosides in red and white Panax ginseng

\begin{tabular}{|c|c|c|c|c|c|}
\hline \multirow[t]{2}{*}{ No. } & \multirow[t]{2}{*}{ Target } & \multicolumn{2}{|l|}{ White Panax ginseng } & \multicolumn{2}{|l|}{ Red Panax ginseng } \\
\hline & & $\begin{array}{l}\text { Concentration (mg/ } \\
\mathrm{kg})\end{array}$ & Calibration curve & $\begin{array}{l}\text { Concentration (mg/ } \\
\mathrm{kg})\end{array}$ & Calibration curve \\
\hline 1 & F1 & 2.8 & $y=2,281,495 x+253,895$ & 66.7 & $y=668,989 x+1,784,117$ \\
\hline 2 & F2 & 1.6 & $y=999,228 x+65,166$ & 14.7 & $y=821,685 x+483,234$ \\
\hline 3 & F3 & 2.5 & $y=5,595,270 x+546,296$ & 7.4 & $y=5,723,388 x+1,685,976$ \\
\hline 4 & F4 & ND & $y=12,424 x+1133$ & 170.0 & $y=16,574 x+1135$ \\
\hline 5 & K & 1.2 & $y=2,450,260 x+116,697$ & 5.8 & $y=2,121,868 x+491,795$ \\
\hline 6 & Mc & 1.4 & $y=2,339,650 x+129,976$ & 9.4 & $y=1,859,636 x+699,869$ \\
\hline 7 & PPT(S) & ND & $y=985,763 x+37,869$ & ND & $y=1,020,181 x+323,826$ \\
\hline 8 & $\mathrm{Rb} 1$ & 26400.0 & $y=93,953 x+12,405$ & 43400.0 & $y=246,459 x+53,362$ \\
\hline 9 & $\mathrm{Rb} 2$ & 2470.0 & $y=161,300 x+39,812$ & 8600.0 & $y=91,018 x+3923$ \\
\hline 10 & Rc & 2840.0 & $y=423,512 x+120,178$ & 5200.0 & $y=353,326 x+9185$ \\
\hline 11 & $\mathrm{Rd}$ & 270.0 & $y=464,148 x+12,722$ & 1270.0 & $y=702,761 x+89,573$ \\
\hline 12 & $\operatorname{Re}$ & 8800.0 & $y=210,640 x+9170$ & 7400.0 & $y=426,878 x+15,761$ \\
\hline 13 & $\mathrm{Rf}$ & 1390.0 & $y=30,371 x+4211$ & 5730.0 & $y=24,456 x+14,001$ \\
\hline 14 & $\operatorname{Rg} 1$ & 11000.0 & $y=533,966 x+29,511$ & 3.7 & $y=937,472 x+139,157$ \\
\hline 15 & $\operatorname{Rg} 2(\mathrm{R})$ & 9.9 & $y=12,172 x+4801$ & 425.0 & $y=15,862 x+2690$ \\
\hline 16 & $\operatorname{Rg} 2(S)$ & 257.5 & $y=16,335 x+1689$ & 980.0 & $y=12,997 x+5095$ \\
\hline 17 & $\operatorname{Rg} 3(R)$ & 1.9 & $y=232,642 x+17,599$ & 360.0 & $y=197,158 x+7162$ \\
\hline 18 & $\operatorname{Rg} 3(S)$ & 8.4 & $y=193,641 x+65,009$ & 700.0 & $y=252,146 x+17,587$ \\
\hline 19 & Rg5 & 6.5 & $y=15,542 x+4013$ & 1140.0 & $y=21,173 x+2450$ \\
\hline 20 & Rg6 & ND & $y=10,369 x+1504$ & 80.0 & $y=18,429 x+592$ \\
\hline 21 & $\mathrm{Rh1}(\mathrm{R})$ & 2.6 & $y=91,155 x+9439$ & 940.0 & $y=321,547 x+30,177$ \\
\hline 22 & $\operatorname{Rh} 1(S)$ & 30.8 & $y=23,186 x+28,575$ & 1600.0 & $y=90,659 x+14,513$ \\
\hline 23 & $\operatorname{Rh} 2(\mathrm{R})$ & ND & $y=522,225 x+1595$ & 2.2 & $y=652,162 x+57,794$ \\
\hline 24 & $\operatorname{Rh} 2(S)$ & ND & $y=707,708 x-8434$ & 7.7 & $y=566,959 x+173,611$ \\
\hline 25 & $X \mathrm{VII}$ & 43.3 & $y=2,844,246 x+4,925,108$ & 147.1 & $y=2,249,081 x+13,234,868$ \\
\hline 26 & Y & ND & $y=2515 x+522$ & ND & $y=2198 x+56$ \\
\hline
\end{tabular}


c

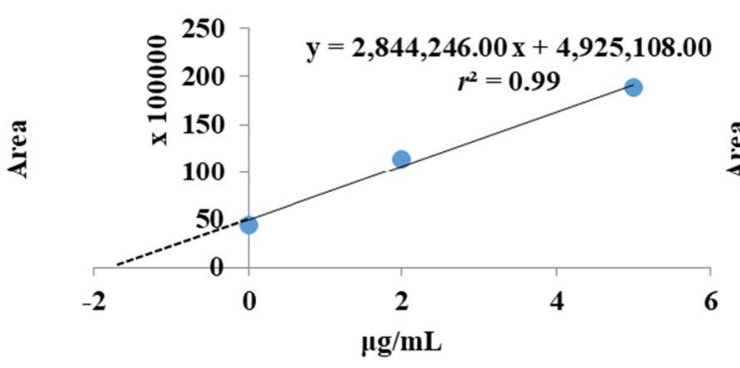

Rg2(S) (QG-II)-WG

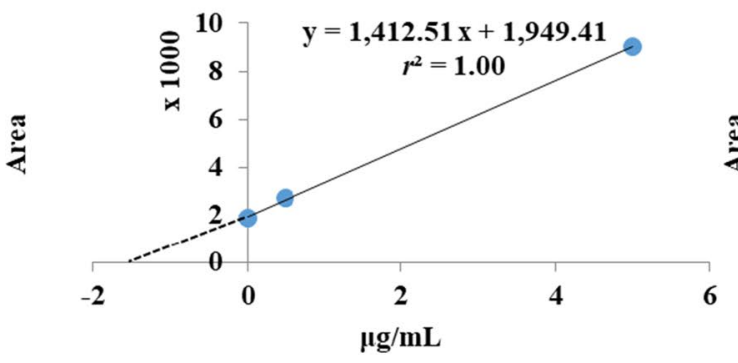

Rd (QG-III)-WG

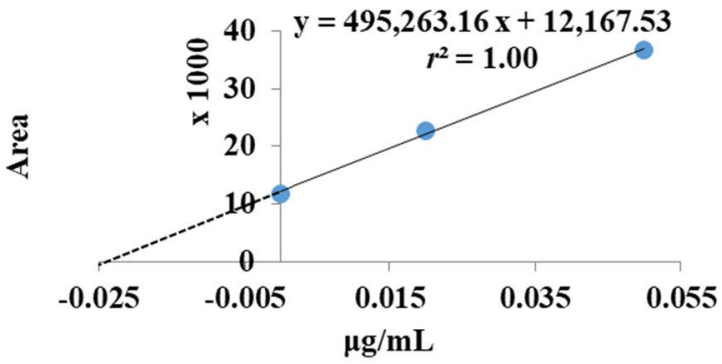

Re (QG-IV)-WG

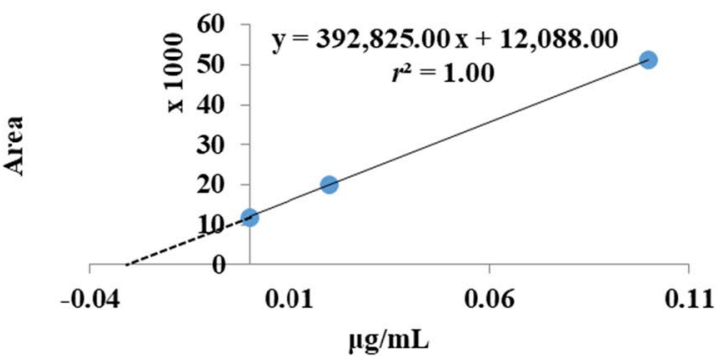

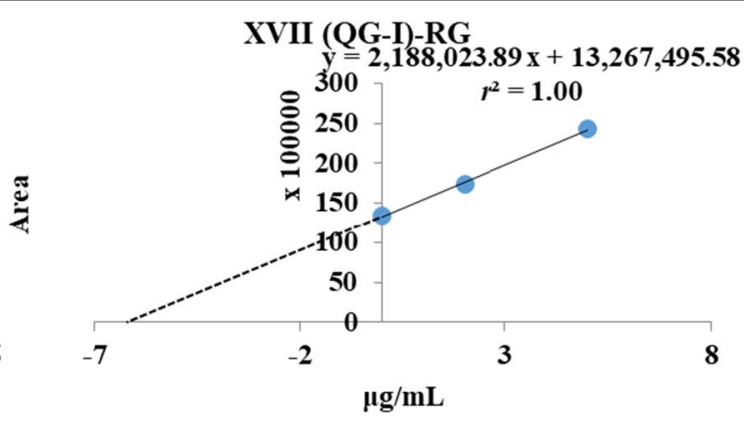

Rg2(S) (QG-II)-RG

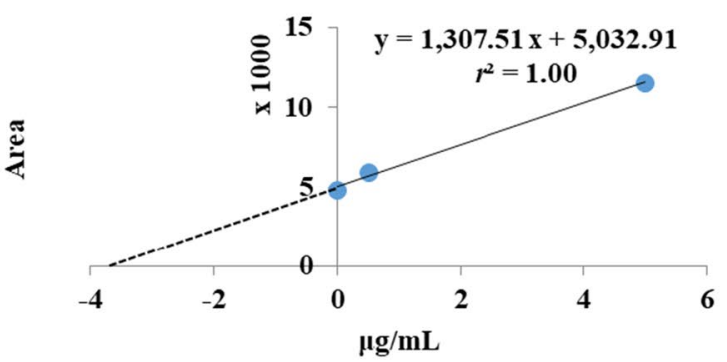

Rd (QG-III)-RG

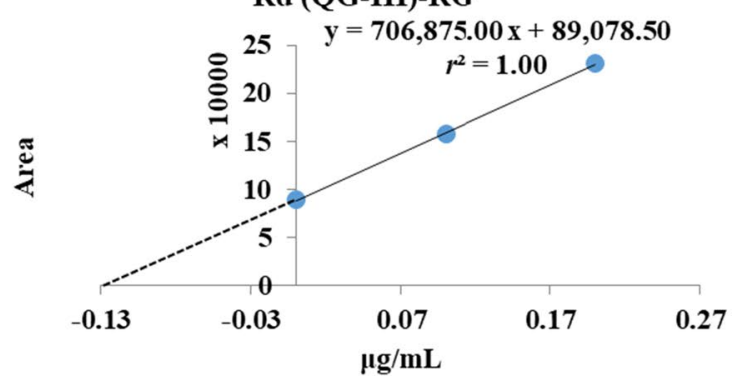

$\operatorname{Re}(\mathrm{QG}-\mathrm{IV})-\mathrm{RG}$

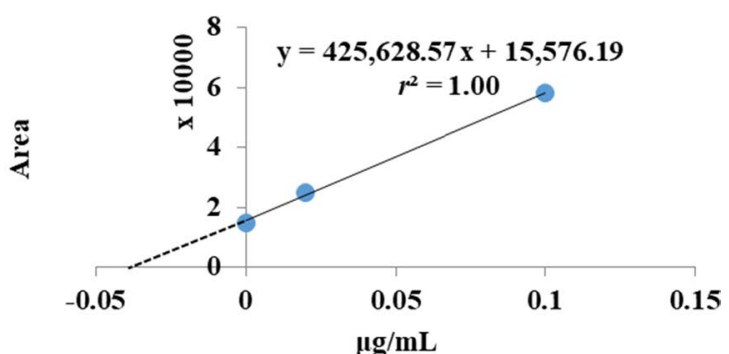

Fig. 3 Representative standard addition calibration curve (WG; white Panax ginseng and RG; red Panax ginseng)

\section{Supplementary information}

The online version contains supplementary material available at https://doi. org/10.1186/s13765-020-00588-w.

Additional file 1: Fig S1. MRM chromatograms of 26 ginsenosides at $10 \mu \mathrm{g} / \mathrm{mL}$ (target ginsenoside is located at middle of chromatogram)
Abbreviations

UHPLC-MS/MS: Ultra-high-performance liquid chromatography-tandem mass spectrometry; MRM: Multiple reaction monitoring; WG: White Panax ginseng; RG: Red Panax ginseng; ESI: Electrospray ionization; QG-I: Quantitation group |: QG-II: Quantitation group || (100 times dilution); QG-III: Quantitation group || (400 times dilution); QG-IV: Quantitation group II (8000 times dilution).

\section{Authors' contributions}

$J L$ performed the experiment, data analysis and interpretation, and wrote the final manuscript. $\mathrm{HJ}$ set up the experimental conditions for the instrumental analysis and performed the data analysis. XY contributed to the design of 
the experimental conditions. EP assisted with the design and conduct of the experiment. JHK and JL supervised the project and revised the final manuscript. All authors read and approved the final manuscript.

\section{Funding}

Funding information is not applicable/No funding was received.

\section{Availability of data and materials}

All data generated or analyzed during this study are included in this published article.

\section{Competing interests}

The authors declare that they have no competing interests.

Received: 1 December 2020 Accepted: 30 December 2020 Published online: 24 January 2021

\section{References}

1. Park SY, Park JH, Kim HS, Lee CY, Lee HJ, Kang KS et al (2018) Systemslevel mechanisms of action of Panax ginseng: a network pharmacological approach. J Ginseng Res 42(1):98-106. https://doi.org/10.1016/j. jgr.2017.09.001

2. Huai-You Wang, Lian-Wen Qi C-ZW and PL. Bioactivity Enhancement of Herbal Supplements by Intestinal Microbiota Focusing on Ginsenosides. Am J Chin Med. 2011;39(6):1103-1115. https://www.ncbi.nlm.nih.gov/ pmc/articles/PMC3624763/pdf/nihms412728.pdf

3. Fuzzati N. Analysis methods of ginsenosides. J Chromatogr B Anal Technol Biomed Life Sci. 2004;812(1-2 SPEC. ISS.):119-133

4. Liu F, Ma N, He C, Hu Y, Li P, Chen M et al (2018) Qualitative and quantitative analysis of the saponins in Panax notoginseng leaves using ultraperformance liquid chromatography coupled with time-of-flight tandem mass spectrometry and high performance liquid chromatography coupled with UV detector. J Ginseng Res 42(2):149-157

5. Le Zhou Q, Zhu DN, Yang XW, Xu W, Wang YP (2018) Development and validation of a UFLC-MS/MS method for simultaneous quantification of sixty-six saponins and their six aglycones: application to comparative analysis of red ginseng and white ginseng. J Pharm Biomed Anal 159:153-165. https://doi.org/10.1016/j.jpba.2018.06.048

6. Xu XF, Cheng XL, Lin QH, Li SS, Jia Z, Han T et al (2016) Identification of mountain-cultivated ginseng and cultivated ginseng using UPLC/Oa-TOF MSE with a multivariate statistical sample-profiling strategy. J Ginseng Res 40(4):344-350

7. Wu W, Sun L, Zhang Z, Guo Y, Liu S (2015) Profiling and multivariate statistical analysis of Panax ginseng based on ultra-high-performance liquid chromatography coupled with quadrupole-time-of-flight mass spectrometry. J Pharm Biomed Anal 107:141-150. https://doi. org/10.1016/j.jpba.2014.12.030

8. Lee J, Shin Y, Lee J, Lee J, Kim BJ, Kim JH (2018) Simultaneous analysis of 310 pesticide multiresidues using UHPLC-MS/MS in brown rice, orange, and spinach. Chemosphere 207:519-526. https://doi.org/10.1016/j. chemosphere.2018.05.116

9. Jo JJ, Cho PJ, Lee S (2018) Simultaneous quantification of 13 ginsenosides by LC-MS/MS and its application in diverse ginseng extracts. Mass Spectrom Lett 9(2):41-45

10. Lv Q, Rong N, Liu LJ, Xu XL, Liu JT, Jin FX et al (2016) Antitumoral Activity of (20 R)- and (20 S)-Ginsenoside Rh2 on Transplanted Hepatocellular Carcinoma in Mice. Planta Med 82(8):705-711

11. Kang S, Song MJ, Min H (2017) Antiviral activity of ginsenoside Rg3 isomers against gammaherpesvirus through inhibition of p38- and JNK-associated pathways. J Funct Foods 2018(40):219-228. https://doi. org/10.1016/j.jff.2017.11.011

12. Cheong JH, Kim H, Hong MJ, Yang MH, Kim JW, Yoo H et al (2015) Stereoisomer-specific anticancer activities of ginsenoside Rg3 and Rh2 in HepG2 cells: disparity in cytotoxicity and autophagy-inducing effects due to 20(S)-epimers. Biol Pharm Bull 38(1):102-108

13. Baek SH, Bae ON, Park JH (2012) Recent methodology in Ginseng analysis. J Ginseng Res 36(2):119-134
14. Xiu Y, Ma L, Zhao H, Sun X, Li X, Liu S (2019) Differentiation and identification of ginsenoside structural isomers by two-dimensional mass spectrometry combined with statistical analysis. J Ginseng Res 43(3):368-376

15. MacCrehan WA, White CM (2013) Simplified ultrasonically- and microwave-assisted solvent extractions for the determination of ginsenosides in powdered Panax ginseng rhizomes using liquid chromatography with UV absorbance or electrospray mass spectrometric detection. Anal Bioanal Chem 405(13):4511-4522

16. Xu XF, Xu SY, Zhang Y, Zhang H, Liu MN, Liu H, et al. Chemical comparison of two drying methods of mountain cultivated ginseng by UPLC-QTOFMS/MS and multivariate statistical analysis. Molecules. 2017;22(5):717

17. Yang H, Lee DY, Kang K Bin, Kim JY, Kim SO, Yoo YH, et al. Identification of ginsenoside markers from dry purified extract of Panax ginseng by a dereplication approach and UPLC-QTOF/MS analysis. J Pharm Biomed Anal. 2015;109:91-104. http://dx.doi.org/10.1016/j.jpba.2015.02.034

18. Yang L, Li CL, Cheng YY, Tsai TH (2019) Development of a validated UPLC-MS/MS method for analyzing major ginseng saponins from various ginseng species. Molecules 24(22):4065

19. Verdu CF, Gatto J, Freuze I, Richomme P, Laurens F, Guilet D (2013) Comparison of two methods, UHPLC-UV and UHPLC-MS/MS, for the quantification of polyphenols in cider apple juices. Molecules 18(9):10213-10227

20. Song H, Song KW, Hong SP (2020) Simultaneous quantification of six nonpolar ginsenosides in white ginseng by reverse-phase high-performance liquid chromatography coupled with integrated pulsed amperometric detection. J Ginseng Res 44(4):563-569. https://doi.org/10.1016/j. jgr.2019.07.002

21. European commission. European Commission. Guidance document on analytical quality control and validation procedures for pesticide residues and anlysis in food and feed (SANTE/11813/2017). 2017

22. Liang J, Chen L, Guo YH, Zhang M, Gao Y. Simultaneous Determination and Analysis of Major Ginsenosides in Wild American Ginseng Grown in Tennessee. Chem Biodivers. 2019;16(7):e1900203

23. Park HW, In G, Han ST, Lee MW, Kim SY, Kim KT et al (2013) Simultaneous determination of 30 ginsenosides in Panax ginseng preparations using ultra performance liquid chromatography. J Ginseng Res 37(4):457-467

24. In G, Seo HK, Park HW, Jang KH. A metabolomic approach for the discrimination of red ginseng root parts and targeted validation. Molecules. 2017:22(3):471

25. In G, Ahn NG, Bae BS, Han ST, Noh KB, Kim CS (2012) New method for simultaneous quantification of 12 ginsenosides in red ginseng powder and extract: in-house method validation. J Ginseng Res 36(2):205-210

\section{Publisher's Note}

Springer Nature remains neutral with regard to jurisdictional claims in published maps and institutional affiliations.

\section{Submit your manuscript to a SpringerOpen ${ }^{\circ}$ journal and benefit from:}

- Convenient online submission

- Rigorous peer review

- Open access: articles freely available online

- High visibility within the field

- Retaining the copyright to your article

Submit your next manuscript at $>$ springeropen.com 